\title{
The Clinical Supervision Process of Nurse Preceptors in Thailand: A Meta-Ethnography Research
}

\section{Chawapon SARNKHAOWKHOM ${ }^{1, *}$ and Ittipaat SUWATHANPORNKUL ${ }^{2}$}

\author{
${ }^{I}$ Faculty of Nursing, Saint Louis College, Bangkok 10120, Thailand \\ ${ }^{2}$ Faculty of Education, Srinakharinwirot University, Bangkok 10110, Thailand
}

('Corresponding author's e-mail: chawapon.s@yahoo.com)

Received: 26 April 2018, Revised: 31 July 2018, Accepted: 25 August 2018

\begin{abstract}
The purpose of this study was to synthesize five graduate theses on nurse preceptors by using the Meta-Ethnography research method. The research findings fell into three steps, as follows: Step 1Preparing for teaching. Nurse preceptors prepared for their clinical supervision with four sub-steps, 1) preparing for readiness, 2) studying and reviewing the clinical practice and clinical supervision guidelines of the organization, 3) planning and designing a clinical supervision model, and 4) making clinical supervision plans; Step 2- Teaching. This step consisted of seven sub-steps, 1) creating familiarity with the learners, 2) clinical practice orientation, 3) clarifying the performance evaluation criteria, 4) preconference, 5) clinical teaching from real situations, 6) post- conference, and 7) improving clinical teaching skills; Step 3- Teaching evaluation. This step consisted of three sub-steps, as follows. 1) selfevaluation by learners, 2) reflection-evaluation by nurse preceptors, and 3) documentation of evaluation by nurse preceptors. Therefore, nursing administrators can implement the results of this research as a process for clinical supervision and develop supervision systems in the nursing organizations to be more effective.
\end{abstract}

Keywords: Clinical supervision, Clinical teaching, Nurse-Preceptor, Meta-ethnography research

\section{Introduction}

Nurse preceptors play important roles in the development of potential newly-graduated nurses and the clinical learning outcomes of nursing students [1]. This is especially true in the case of newlygraduated nurses or novices during the role transition from nursing students to professional nurses who have some very independent roles and more responsibilities, such as nursing services, nursing implementation, and academic roles; all these things are far more than newly-graduated nurses are capable of [2]. Newly-graduated nurses, or 'novices', have ability as beginners who have very little experience and practice, including academic knowledge; welding theory into action is not enough to work as a professional [3]. Therefore, novices normally face difficulties in finding success as professional nurses at first; they also have to adapt themselves into the organizations, working systems, and with colleagues, and normally feel uneasy, stressful, and confused, and lose confidence and find themselves to be less useful [4]. These negative feelings could affect the professional nurses, nursing organizations, and the people who receive their services; the rates of resignation increase and, therefore, novices should have systems in order to develop themselves to work as professionals effectively, increase their performance standards, and adapt themselves to the work, which will result in a high quality of nursing service [5].

A novice potential development system which has been used globally is the preceptorship system, which was developed by Vance [6]; this is an idea of managing human resources in the organization, focusing on the relationships between coworkers, which helps staff to be satisfied with the job and improve themselves to be professionals. The system is very popular, and has resulted in an increase in 
practicing skills and academic knowledge [7]. To success in a job as a nurse, at least one preceptor who has experience and more knowledge is needed, who will have to supervise clinical processes and work with novices as "nurse preceptors" [8].

Recently, there have been many studies from within and outside the country concerning nurse preceptors, especially, qualitative studies that focus on the experiences of nurse preceptors, the work that they have been doing, and their clinical supervision processes [5]. However, there has never been a study in the way of research synthesis on the clinical supervision processes of mentors, which could help to bring every problem to the fore as a synthesis, which could be far more useful than summarizing each thesis; the process includes quantitative research synthesis and qualitative research synthesis [9]. A qualitative research synthesis that has been very popular is Meta-Ethnography Research [10,11].

From a review of the literature, most studies of nurse preceptors in Thailand have been in the form of qualitative research. The results of the studies were varied from the contexts, and therefore have limited use in actual work. Therefore, a research synthesis will help bring out the useful points for professional nurses, hospital supervisors, or nursing administrators to use in the development of clinical supervision processes or clinical supervision systems. The purpose of this study was to synthesize graduate theses on nurse preceptors by using the Meta-Ethnography research method. This study focuses on the synthesis process of clinical supervision of nurse preceptors using the clinical supervision process of Kittiratchada and Wanichpanjapol [12], which includes: 1) Planning process, 2) Organizing process, 3) Leading process, 4) Controlling process, and 5) Assessing process, as a tentative theory to synthesize the clinical supervision processes of nurse preceptors.

\section{Materials and methods}

This study was a qualitative research synthesis of the clinical supervision processes of nurse preceptors using the Meta-Ethnography research method of Noblit \& Hare [10], which represent seven processes: 1) Getting started, 2) Deciding what is relevant to the initial interest, 3) Reading the studies, 4) Determining how the studies are related, 5) Translating the studies into one another, 6) Synthesizing the translation, and 7) Expressing the synthesis. The studies that were included in this research synthesis were graduate theses on the experiences of nurse preceptors from five books from the years 2007 - 2016 taken from the theses of ten books, as per the following research methods;

1. Searching the graduate thesis lists of nurse preceptors from the Thai Library Integrated System (ThaiLIS) database, which is the database of the complete research document collection from universities around Thailand, by using "Clinical supervision", "Clinical teaching", "Nurse-Preceptor", and "Supervision process" as keywords, giving 36 books.

2. Collecting research results from the search in the first step and selecting research collection from the criteria, defined as: 1) Qualitative research on nurse preceptors' experiences, and 2) the research studies must have been conducted in Thailand, and completed between the years 2007 - 2016. In this step, we found the theses from ten books that met the criteria.

3. Evaluating the research quality by using a research quality assessment form developed by evaluation in the dimension of content, research methodology, and the utilization of research results, which was evaluated into four levels as "very good", "fair", "moderate", and "should improve", to be used in quality assessment. The research quality assessment form had to clarify and quantify the quality according to five experts, and the results of the research quality had to be at least $85 \%$ to be used to synthesize qualitative research by Meta-Ethnography Research. In this step, we found theses that met the criteria in five books, and theses that did not meet the criteria were excluded.

4. Analyzing the data by interpretation of the research in the terms of Meta-Ethnography research, following the concept of Noblit and Hare [10] for the synthesis of the clinical supervision processes of nurse preceptors. The interpretation was divided into three types: 1) Reciprocal translation, by bringing the same or similar research or metaphors directly to other research, 2) Refutation translation, by bringing together conflicting issues, where it could not be interpreted as reciprocal translation to defy a relationship but in a line of reasonable confliction, and 3) Line of argument, to present the point of contention related to the reference, the conclusion, and the overall picture by synthesis. However, we 
would like to give an example of data analysis by interpreting the results of the research, as shown in Table 1.

Table 1 Example of interpretation and translation.

\begin{tabular}{llll}
\hline Reciprocal translation & Refutation translation & \multicolumn{1}{c}{ Line of argument } & Results of translation \\
\hline & & $\begin{array}{l}\text { 1. Preparing the body } \\
\text { before teaching }\end{array}$ & \\
Preparing for advice & $\begin{array}{l}\text { 1. Body preparation } \\
\text { 2. Preparing the } \\
\text { 2. Mind preparation }\end{array}$ & $\begin{array}{l}\text { information } \\
\text { 3. Preparing the nursing } \\
\text { procedure techniques }\end{array}$ & \\
& &
\end{tabular}

As in Table 1, defying reciprocal translation, a comparison of metaphors was derived from the findings of consistent research. In each research, a constant was the "Preparation for advice"; the refutation translation was a comparison of the findings from each research of the differentiate of results "Body and Mind preparation". For the line of argument, the findings from synthetic research linked the results of the research, "Preparing the body before teaching", "Preparing the information" and "Preparing the nursing procedure techniques". So, the interpretation of the three forms is to achieve the synthesis "Prepare for readiness".

5. Summarizing and discussing the results of this study.

\section{Results and discussion}

The research synthesis findings of the clinical supervision processes of nurse preceptors were divided into three major steps: 1) Preparing for teaching, 2) Teaching, and 3) Teaching Evaluation.

\section{Step 1: Preparing for teaching}

After being assigned by supervisors to serve as nurse preceptors, one of their missions is the clinical supervision of new graduates or novice nurses who work in the department; before the nurse can conduct clinical supervision, they must prepare using four steps, as follows.

1.1) Prepare for readiness. Nurse preceptors must prepare their body and knowledge before clinical teaching and supervision to gain confidence after being entrusted to serve as nurse preceptors with knowledge of diseases, nursing procedures, nursing care, nursing techniques, and new technology for patient care by studying from the internet, documents, and books, including work instruction, and discussing with doctors or experienced nurses, to be ready to supervise.

1.2) Study and review clinical practice and clinical supervision guidelines of the organization. Nurse preceptors must do this before supervising or teaching to make the clinical supervision process smooth and to gain understanding of the organizational structure, including the management system in various matters of the organization or its agencies, for novice orientation and supervision.

1.3) Plan and design a clinical supervision model. When nurse preceptors are prepared to clinically supervise novices, are in charge of the preparation of knowledge and education, and have reviewed the clinical practice guidelines of the organization, they then plan and design which kind of supervision will be used in clinical supervision, considering which instruction methods and supervision plans will be effective in clinical supervision allowing for more understanding and effectiveness of the job, which achieves the purpose of the learning.

1.4) Make clinical supervision plans. Before the nurse preceptors conduct clinical supervision, to be able to supervise as planned, it is considered to be one of the preceptor's duties to plan and present that plan to supervisors after completing their duties. 


\section{Step 2: Teaching}

After the first step, the preparation step, is completed, nurse preceptors need to use the plans and preparation to supervise novices as assigned. Step 2 consists of seven steps, as follows:

2.1) Create familiarity with the learners. Before conducting clinical supervision, start to get familiar with the novices and nursing students, build relationships with simple conversations, and create a nice atmosphere to make for relaxing and free supervision by asking typical questions about the nursing job to understand the background and attitudes of the novices and nursing students in order to plan the supervision.

2.2) Clinical practice orientation. When the nurse preceptors are familiar with newly-graduated nurses or nursing students by asking typical questions, nurse preceptors will provide orientation for the operation by introducing the general contents of department storage facilities, regulations in the operation, the times for work operation and the lifestyle during operation for newly-graduated nurses or nursing students in the custody of nurse preceptors, including increasing familiarity with the patients of the department, and feeling part of the department.

2.3) Clarify the performance evaluation criteria. After creating familiarity and providing orientation for clinical practice, nurse preceptors need to provide details of the evaluation criteria and evaluation period to novices to help them prepare for their own performance evaluation.

2.4) Pre-conference. Before processing any duty of clinical supervision, nurse preceptors shall play a leading role in counseling sessions and ask for knowledge to stimulate novices. The questionnaire asked about patients to provide theoretical learning, along with clinical practice, and will help in observing the behavior, attention, and enthusiasm of novices to be used in the evaluation of performance and to improve their knowledge, understanding, and clinical skills.

2.5) Clinical training from real situations. The clinical supervision of nurse preceptors uses training from real situations or on-the-job training. Nurse preceptors assign responsibilities, duties, and patient care to novices, and novices are expected to complete their preparation and seek information of their assignments one day in advance of the training session before starting work on wards the next day. Then, nurse preceptors will teach novices from real situations, along with real clinical practice and real experiences. During clinical training, nurse preceptors need to observe the behavior of novices who practice on wards so as to make decisions in the evaluation of performance, improve teaching techniques, and arrange some experience to gain more clinical processes and knowledge.

2.6) Post-conference. After operation, newly-graduated nurses or nursing students must always have a post-conference. The post- conference consultation is about reviewing the knowledge of newlygraduated nurses or nursing students of patients of the department they see in the operation of their work that day and their experiences of clinical patients of the department, including patients with interesting cases. Nurse preceptors discuss case studies for coordinating learning for newly-graduated nurses or nursing students. Nurse preceptors observe the behavior, heedfulness, interest, and enthusiasm of newlygraduated nurses or nursing students in operation in patient departments during consultation operation as well as during pre-operation.

2.7) Improve teaching skills. After nurse preceptors observe practices, including assessment of the knowledge, understanding, and clinical practice skills of novices, they shall improve their teaching skills to create clearer explanation and use experiences in teaching as preceptors.

\section{Step 3: Teaching Evaluation}

This step includes three procedures: 1) self-evaluation by learners, 2) reflection-evaluation by nurse preceptors, and 3) documentation of evaluation by nurse preceptors, as follows;

3.1) Self-evaluation by learners. After completing supervision, novices or nursing students will evaluate themselves by discussing and asking about what they have learnt, what they wanted to develop or gain about clinical processes or techniques, and their feelings after completing the task.

3.2) Reflection-evaluation by nurse preceptors. Newly-graduated nurses or nursing students who complete their training on the ward have completed their self-evaluation. Nurse preceptors perform a reflection on the results of the evaluation of newly-graduated nurses or nursing students who performed the on-the-job training based on self-evaluation data. The results of the evaluation after the clinical 
supervision are notified to newly-graduates nurses and nursing students who practice on the ward, who can use it to improve and self-improve, as per instructions.

3.3) Documentation of evaluation by nurse preceptors. After nurse preceptors have given the opportunity to newly-graduated nurses and nursing students who practice on the ward to self-evaluate, nursing preceptors reflected the results of the evaluation to them. Nurse preceptors sign the evaluation record. The signed results are kept as evidence of the evaluation of the performance on the ward. The results of the clinical evaluation are then communicated to relevant stakeholders and relevant agencies.

\section{Discussion}

After synthesis of the clinical supervision processes of nurse preceptors, we found that the clinical supervision process included three steps: Step 1- Preparing for teaching, Step 2- Teaching, and Step 3Teaching Evaluation. These three steps include procedures as follows; 1) Step 1 has four procedures- 1.1) prepare for readiness, 1.2) study and review of clinical practice and clinical supervision guidelines of the organization, 1.3) plan and design a clinical supervision model, and 1.4) make clinical supervision plans. 2) Step 2 has seven procedures- 2.1) create familiarity with the learners, 2.2) clinical practice orientation, 2.3) clarify the performance evaluation criteria, 2.4) pre-conference, 2.5) clinical teaching from real situations, 2.6) post-conference, and 2.7) improve clinical teaching skills. 3) Step 3 has 3 procedures- 3.1) self-evaluation by learners, 3.2) reflection-evaluation by nurse preceptors, and 3.3) documentation of evaluation by nurse preceptors. From every step of the clinical supervision process as mentioned, it was found that both consistency and difference from the concept of the clinical supervision process, as stated by Kittiratchada and Wanichpanjapol [12], that the clinical supervision process includes five procedures, which are 1) the Planning process, which will define the philosophy, principles, concepts, goals, and purpose of supervision, including annual plans, monthly plans, the beginning of the supervisory system to be used in practice, and the performance evaluation, which is in line with the results of the synthesis in step 1, the preparation step that nurse preceptors must have knowledge to prepare about diseases, patient care, and clinical techniques by studying from the Internet, various documents, or related books, work instruction, the supervision process of the organization, guidelines, and write the clinical supervision plan before teaching or supervision.

The results of the synthesis in step two is in teaching. Nurse preceptors begin clinical supervision by creating familiarity with learners and establishing relationships between students and preceptor. Then, nurse preceptors provide orientation for the patient ward, explain about the performance appraisal, and describe the criteria for performance evaluation and the time to evaluate the performance. Then, a preconference for assignment of duties of nursing care to newly-graduated nurses and nursing students who practice on the ward and clinical work from the job site is given. After that pre-conference, nurse preceptors observe the behavior and assess the knowledge, understanding, and skills of newly-graduated nurses and nursing students who practice on the ward. The data from the observations of new nursing graduate behavior and the students who practice on the ward are used to improve teaching to the new nurse graduates and students who have been trained on the ward to have more knowledge, understanding, and skills. This is consistent with step 2 and 3 of the clinical supervision process based on the concept of Kittiratchada and Wanichpanjapol [12], which is step 2, the Organizing process, to establish criteria and terms of work, provide resources for the operation, establish relationships, divide tasks, assign tasks, and provide contact coordination. Step 3, the Leading process, promotes performing demonstrations, counseling, and encouraging the workers to develop and to continue working.

Step 3, the results of the synthesis, is the evaluation of the teaching. The post-evaluation of nurse preceptors begins with newly-graduated nurses and students who practice on the ward performing selfevaluation. Then, nurse preceptors record the results of the evaluations by signing. The signed results are kept as evidence in the assessment of performance on the ward and the results of the clinical evaluation are then communicated to relevant stakeholders and relevant agencies. In the evaluation stage, this result corresponds to step 5, the Assessing process. This is an evaluation of performance, performance measurement, and diagnostic performance [12].

Therefore, the clinical supervision process is based on the concept of Kittiratchada and Wanichpanjapol [12] and comply with the synthesis results over four steps; however, there are steps that 
are not consistent with the synthesis result, namely Step 4, the Controlling process. This is a convenient procedure for the operator to correct warnings when the operation is erroneous, including regulations of work. It is possible that, in the process of controlling this operation, nurse preceptors were not responsible for the implementation of regulations. Regulations have a role to play for teacher, advisers, and assessors [13].

However, determining regulations for the operation is the role of the management organization and nursing administrators [14], and facilitate the operator to correct warnings when the wrong operation is performed, and nursing preceptors are routinely monitored during clinical supervision. It is important to keep in touch with instructors, newly-graduated nurses and nursing students who practice on the ward. The fourth step of the clinical supervision process is based on the concept of Kittiratchada and Wanichpanjapol [12]. There are some similarities with the results of the clinical nurse preceptor supervision synthesis process. This is why there are some parts that are not consistent with the fourth step of Kittiratchada and Wanichpanjapol [12].

\section{Conclusions}

Nurse preceptors have the important roles to play for development of potential newly-graduated nurses and the clinical learning outcomes of nursing students. This meta-ethnography result was founded that the clinical supervision processes of nurse preceptors consists of three major steps started with the preparation of teaching follow by teaching and teaching evaluation in the last steps. All of these steps, nursing administrators can implement and apply these steps as guidelines for the clinical supervision of nurse preceptors or to develop a supervision system for the clinical work of mentors in organizations to be more effective. The study also found that the clinical supervision process of nurse preceptors is a process that is undertaken by nurse preceptors with a lack of support from organizations at various stages. Therefore, the support of organizations in the clinical supervision process of nurse preceptors should be studies, in order to be used as information to improve and promote the support of organizations in the development of the clinical supervision system.

\section{Acknowledgements}

The authors would like to thank the faculty members of the Department of Educational Measurement and Research, Faculty of Education, Srinakharinwirot University, and the Faculty of Nursing, Saint Louis College, for their support.

\section{References}

[1] PE Ngaiyaye, T Bvumbwe and CM Chipeta. Using preceptors to improve nursing students' clinical learning outcomes: A Malawian students' perspective. Int. J. Nurs. Sci. 2017; 4, 164-8.

[2] C Gerrish. Fumbling along. Nurs. Times. 1990; 86, 35-7.

[3] P Benner. From novice to expert. California, Addison-Wesley, 1984, p. 10-5.

[4] T Sanbudda. 2003, Experience of New Graduates in General Hospitals. Master Thesis. Chulalongkorn University, Bangkok, Thailand.

[5] S Sammawaja. Implementing a system in nursing instructors. Ramathibodi Nurs. J. 2000; 6, 52-67.

[6] CN Vance. The mentor connection. J. Nurs. Adm. 1982; 12, 7-13.

[7] S Komartaj. 2006, The Effect of Implementing Nurse Preceptors on Nursing Practice of New Nursing Graduation. Master Thesis. Chulalongkorn University, Bangkok, Thailand.

[8] AJ Dubrin. Applying Psychology: Individual and Organizational Effectiveness. Prentice Hall, New Jersey, 2000, p. 30-7.

[9] N Wirajchai. Meta-Analysis. Nissin Advertising Group, Bangkok, 1999, p. 10-21.

[10] B Meksritongkam. Meta-ethnographic research: Qualitative research synthesis. J. Nurs. Sci. 2009; 36, 77-85.

[11] GW Noblit and RD Hare. Meta-Ethnography: Synthesizing Qualitative Studies. Sage Publications, Newbury Park, 1988, p. 19-32. 
[12] S Kittiratchada and V Wanichanjapol. Nursing Administration to Quality Supervision. Sam Charoen Panich, Bangkok, 2008, p. 21-36.

[13] CK Hall. Role functions of staff nurse preceptors for undergraduate pre-licensure nursing students. J. Nurs. Edu. Prac. 2016; 6, 19-30.

[14] B Sresatitrakul. Leadership and Strategy for Managing Nursing Organization in the $21^{\text {st }}$ Century. Chulalongkorn University Press, Bangkok, 2008, p. 132-48. 